\title{
Research on Financing Efficiency of Technology-Based SMEs Based on DEA-Malmquist-Tobit Method
}

\author{
Lisheng Pan ${ }^{1, a}$,Dan $\mathrm{Li}^{2, \mathrm{~b}}$ \\ ${ }^{1}$ School of Management, Hefei University of Technology, Hefei, Anhui, China \\ ${ }^{2}$ School of Management, Hefei University of Technology, Hefei, Anhui, China \\ apanlish@sina.com, ${ }^{b}$ lidan_super@163.com \\ *Dan Li
}

Keywords: Technology-based enterprise, Financing efficiency, DEA-Malmquist-Tobit method.

\begin{abstract}
As the most dynamic emerging force in China's economic development, science and technology-oriented SMEs cannot develop without the improvement of financing efficiency. This paper builds a financing efficiency evaluation index system that conforms to the industry characteristics, and uses the panel data of 705 technology-based SMEs in 2014-2018 as a research sample. The DEA model and the Malmquist index are used for static and dynamic analysis of financing efficiency. And the Tobit model is used to explore the internal factors of corporate financing efficiency in order to find a path to efficiency. The research results show that the overall level of financing efficiency of SMEs is not high, and it shows a downward trend during the sample period. The low efficiency of pure technology is the main factor that makes the financing efficiency of enterprises difficult to improve. Further research has found that the profitability and operational capability of enterprises have a positive effect on financing efficiency. The excessive debt level of enterprises will inhibit the improvement of financing efficiency, and the development capability of enterprises will not have a significant impact on financing efficiency.
\end{abstract}

\section{我国科技型中小企业融资效率研究 基于DEA-Malmquist-Tobit方法

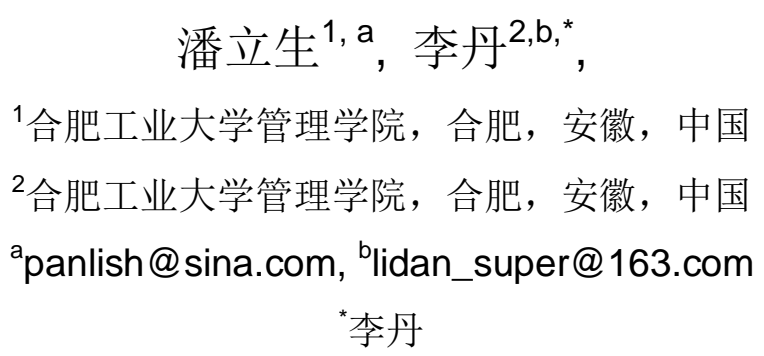

关键词：科技型企业；融资效率；DEA-Malmquist-Tobit方法

中文摘要. 作为我国经济发展中最具活力的新兴力量, 科技型中小企业的健康发展离不开融 资效率的提升。本文通过构建符合行业特征的融资效率评价指标体系, 以705家在新三板挂牌 的科技型中小企业2014-2018年面板数据为研究样本, 运用DEA模型和Malmquist指数对其融 资效率进行静态和动态分析, 并引用Tobit模型探究企业融资效率的内部影响因素以寻求效率 的提升路径。研究结果表明：我国新三板市场中科技型中小企业融资效率整体水平不高, 且 在样本期内呈下降趋势, 纯技术效率低下是导致企业融资效率难以提升的主要因素。进一步 
研究发现: 企业的盈利能力和营运能力对融资效率具有正向促进作用, 企业过高的负债水平 会抑制融资效率的提高，企业的发展能力并不能对融资效率产生明显影响。

\section{1. 引言}

科技创新是引领国家经济发展的第一动力。加快建设创新型国家, 推进产业转型升级, 加大对中小企业科技创新的支持力度，是十九大报告的一个重要内容。目前，我国正处于经 济结构的调整转型时期, 同时也处在产业变革与新科技革命的历史交汇期, 创新已然成为我 国经济发展新的增长极。作为我国多层次资本市场体系的重要组成部分, 新三板市场中的科 技型中小企业近几年迅猛发展，成为我国经济发展中最具活力的新兴力量。然而，在规模快 速发展的同时，科技型中小企业由于其自身风险高、运营成本高以及资本市场体系和融资担 保制度发展不够完善等原因，正面临着融资方式单一、融资成本高等融资难题。无论是加大 科技型企业自主研发力度还是优化调整企业的组织结构, 都需要充沛的资金流和高效的资金 运转效率作支撑。因此, 探究我国新三板市场中科技型中小企业的融资效率现状, 运用 DEA-Malmquist方法动态分析近三年融资效率的变动情况并寻求效率的提升路径, 这对于科 技型中小企业健康成长具有重要意义。

\section{2. 文献研究述评}

\section{1 融资效率的内涵}

“融资效率”这一概念最早是由国内学者曾康霖（1993） ${ }^{[1]}$ 提出，他认为融资的效率是企 业选择直接融资或间接融资方式的重要影响因素。宋文兵（1988） [2]首次从经济学的角度对 融资效率进行了实质性分析, 将融资效率分解为交易效率和配置效率。前者反映企业融通资 金的能力，即衡量企业能否以最低的成本获取资金。后者反映企业对融入资金的使用能力, 即衡量企业能否将有限的资金发挥最大效用。程守红（2003） ${ }^{[3]}$ 认为企业的融资效率可以用 在一定的时间和空间下融通资金的投入产出比进行衡量。高学哲（2005） [4]指出，融资效率 体现在为企业带来收益的融资能力, 将融资效率定义为企业效益与资本成本之比。

\section{2 融资效率相关文献研究}

基于对融资效率概念界定的基础上，国内外学者对于企业融资效率的实证分析展开了广 泛研究。朱冰心（2005） [5]运用模糊综合评价法对浙江中小企业的融资效率进行实证分析, 研究表明中小企业的融资秩序符合金融豚食理论，且实证结果表明企业内源融资的效率高于 外源融资效率。汪涛（2014）[6]较早地使用DEA数据包络分析法测算新三板挂牌企业的融资 效率，从时间、行业和非有效性三个层次对企业的融资效率展开综合评价分析。李欣（2015） [7]选取2011-2013年我国科技型中小企业面板数据为研究样本, 通过对融资效率的动态连续分 析发现，多数企业单纯通过扩大规模或多元化生产来提高效率，而并不注重公司核心技术的 提高和自身管理体制的完善, 内生性增长不足是科技型企业融资效率普遍偏低的重要原因。 修国义和李岱哲（2016） ${ }^{[8]}$ 采用DEA-Malmquist指数法对20家新三板科技型中小企业进行实证 研究，分析发现技术效率过低是导致科技型企业融资效率偏低的主要原因。沈忱（2017）[9] 选择在2015年有过定向融资行为的101家新三板企业为研究对象, 运用DEA三阶段法对融资效 率进行比较研究，分析发现新三板企业融资效率偏低的原因主要是规模效率偏低。

通过梳理前人对于融资效率相关的文献研究，可以发现学术界对于融资效率的内涵并没 有明确的概念界定，比较公认的观点是用融通资金的投入产出比衡量企业的融资效率。对于 研究方法的选择, 多数学者倾向DEA数据包络法研究企业的融资效率, 选取的研究对象大多 集中于新三板中小企业整体, 缺乏对科技型中小企业的深入研究, 忽视了行业的差异性对融 资效率的影响。因此, 本文选取新三板市场中的科技型中小企业作为研究对象, 考虑该行业 
具有高风险、高收益的特点, 合理构建具备行业特点的投入产出指标体系, 运用DEA模型和 Malmquist指数法对科技型中小企业融资效率分别进行静态和动态分析, 探究观察期内企业融 资效率的变动趋势及原因，并引用Tobit模型对融资效率影响因素实证分析。

\section{3. 研究设计}

\section{1 研究方法}

\subsubsection{DEA模型}

数据包络分析法 (Data Envelopment Analysis, DEA)由美国运筹学家Charnes和Cooper于 1978年提出, 它是一种基于多投入多产出视角测定同质决策单元相对效率的数学规划方法。 DEA模型将每个企业视为单个决策单元 (DMU) , 利用非参数线性规划模型将决策单元的投 入产出数据集合构建一个效率前沿面, 通过观察各决策单元是否落在效率前沿面上判断DEA 是否有效。由于该评价方法不用事先对各项指标赋予权重, 更具客观性, 因而在企业融资效 率的研究中得到广泛应用。

固定规模报酬模型（CCR）和可变规模报酬模型（BCC）是DEA方法中两个基本模型。 其中, CCR 模型假定规模报酬不变, 即企业规模的扩大或缩减都不会对企业融资效率产生影 响, 这种前提假设在具体的实际情况中并不适用。而BCC模型对此进行了改进, 在规模报酬 可变的前提下评价企业的融资效率，将决策单元的综合效率值 (TE) 分解为规模效率值 (SE) 和剔除规模效率影响后的纯技术效率值（PTE）。BCC模型的构建过程如下：假设共有 $\mathrm{n}$ 个决 策单元 $D M U_{j}(\mathrm{j}=1,2, \ldots, \mathrm{n})$, 每一个决策单元 $(D M U)$ 均有 $\mathrm{m}$ 种投入和 $\mathrm{s}$ 种产出, 对于第 $\mathrm{j}$ 个决策 单元 $D M U_{j}$ 其投入向量为 $X_{j}=\left(X_{1 j}, X_{2 j}, \ldots \ldots, X_{m j}\right)^{T}$, 产出向量为 $Y_{j}=\left(Y_{1 j}, Y_{2 j}, \ldots \ldots, Y_{s j}\right)^{T}$, 由此构建的生 产可能性集合为:

$$
T=\left\{(X, Y) \mid \sum_{j=1}^{n} X_{j} \gamma_{j} \leq X, \sum_{j=1}^{n} Y_{j} \gamma_{j} \leq Y, \sum_{j=1}^{n} \gamma_{j} \leq 1, \gamma_{j} \geq 0, j=1,2, \ldots \ldots, n\right\}
$$

根据上述集合构建的BBC模型具体如下:

$$
\left\{\begin{array}{c}
\min \left[\theta-\left(\sum_{i=1}^{m} s_{i}^{-}+\sum_{t=1}^{s} s_{t}^{+}\right)\right] \\
\sum_{j=1}^{n} X_{i j} \gamma_{j}+s_{i}^{-}=\theta X_{i j 0}, i \in(1,2, \ldots \ldots, m) \\
\sum_{j=1}^{n} Y_{t j} \gamma_{j}-s_{t}^{+}=Y_{t j 0}, t \in(1,2, \ldots \ldots, s) \\
\theta, \gamma_{j}, s_{i}^{-}, s_{t}^{+} \geq 0, j=1,2, \ldots \ldots, n
\end{array}\right\}
$$

如果 $\theta^{*}=1$ 且 $S_{i}^{-*}=0, S_{t}^{-*}=0$, 表明该决策单元DEA有效, 如果不满足上述条件, 表明该决 策单元非DEA有效, 存在可以改进的空间。

\subsubsection{Malmquist指数}

DEA模型主要适用于评价同一时期内各个决策单元的相对效率，对于探究不同时期内决 策单元的效率变化趋势, 则可以引入Malmquist指数进行动态分析。该方法建立在DEA模型的 基础之上, 利用Shephard距离函数的比率探究企业的全要素生产率（tfpch）在样本期间内的 动态变化。Malmquist指数将全要素生产率分解为技术效率指数 (effch) 和技术进步指数 (techch)，而技术效率指数在规模报酬可变的前提下进一步分解为纯技术效率指数（pech） 和规模效率指数 (sech), 从而更为直观系统地反映全要素生产率变化的具体原因和特征趋 势, 为后续寻找提升决策单元的效率路径提供理论分析依据。其中, 基于规模报酬可变前提 下的Malmquist指数分解公式如下:

$$
\text { tfpch }=e f f c h \times t e c h c h=p e c h \times \sec h \times t e c h c h
$$

从构成全要素生产率的各项指标来看，若某项指标大于 1 ，则表明从 $\mathrm{t}$ 时期到 $\mathrm{t}+1$ 时期，该 项指标对应的效率提高; 若某项指标小于 1 , 则表明从 $\mathrm{t}$ 时期到 $\mathrm{t}+1$ 时期, 该项指标对应的效率 降低; 若等于1, 则表明该项指标对应的效率不变。 


\subsubsection{Tobit回归模型}

Tobit回归模型是一种将因变量限制在一定范围内的样本选择模型, 它解决了因变量不连 续导致的计算难题。由于本文在探究DEA模型中技术效率的影响因素时, 其第一步测算出的 技术效率值在 $0 \sim 1$ 之间, 融资效率值被正向截断。在此情景下, 采用普通的最小二乘法对模型 回归, 会导致参数估计有偏且不一致。因此, 本文采用Tobit计量经济学模型对融资效率的影 响因素进行探究, 保证论证结果的科学性。

\section{2 指标体系构建}

本文基于投入和产出的视角测度企业的融资效率，在运用DEA模型对企业融资效率进行 评价分析时, 选取不同的投入产出指标会对评价结果产生重要影响。因此, 在参考相关文献 的基础上，考虑了科技型中小企业所具有的高投入、高技术、高风险和高收益的特点，本文 最终选取 3 项投入指标和 4 项产出指标构建具有行业特征的科技型企业融资效率评价指标体 系，指标构建具体如表1所示。

\section{2 .1 投入指标的选取}

（1）资产总额：该指标代表企业所拥有或所控制的预计能给企业带来经济利益的资源总 和, 反映了企业整体规模的大小。规模越大的企业, 筹资能力相对越强, 因此该指标一定程 度上代表了企业的融资能力。

（2）资产负债率：该指标是企业负债总额在资产总额中所占的比例，反映企业的资本结 构，将该指标纳入评价指标体系可以从资本结构的层面评价融资效率。

（3）营业总成本：该指标是指企业用于日常生产经营活动中所投入的成本，对企业的收 入与利润产生影响，反映了企业对于融通资金的实际运用效率。

3.2 .2 产出指标的选取

（1）净利润：该指标直接反映了企业经营活动收益情况，是企业经营成果的重要指标。

(2) 净资产收益率：该指标可以反映企业通过运用自有资本获得收益的能力, 净资产收 益率越高，说明企业对于自有资本的利用效率越高。

（3）营业总收入：该指标直观反映出企业融入资金的产出情况。营业收入是企业获得持 续稳定成长的源泉，因此该指标在一定程度上也反映出企业的成长状况和发展能力。

(4) 无形资产增加额: 科技型中小企业的产出除了转化为营业总收入外，其余产出体现 在无形资产之中。无形资产增加额在一定程度上量化了科技型企业的研发成果, 代表了企业 的技术创新能力。

表1 DEA模型指标构建及其计算方式

\begin{tabular}{|c|c|c|}
\hline 指标类型 & 指标名称 & 计算方式 \\
\hline \multirow{3}{*}{ 投入指标 } & 资产总额 & 资产总额 \\
\cline { 2 - 3 } & 资产负债率 & 负债总额/资产总额 \\
\cline { 2 - 4 } & 营业总成本 & 营业总成本 \\
\hline \multirow{4}{*}{ 产出指标 } & 净利润 & 净利润 \\
\cline { 2 - 4 } & 净资产收益率 & 净利润/[(期初所有者权益+期末所有者权益 $) / 2]$ \\
\cline { 2 - 4 } & 营业总收入 & 营业总收入 \\
\cline { 2 - 3 } & 无形资产增加额 & 期末无形资产一期初无形资产 \\
\hline
\end{tabular}

\section{3 数据来源及处理}

\subsection{1 样本选取}

本文依据证监会划分的行业分类标准，选取2014-2018年在新三板上市的科技型中小企业 作为研究对象, 研究企业融资效率在观察期内的变化趋势及原因分析。样本的笁选过程如下:

（1）选取在证监会行业分类标准中划分为 “信息技术行业” 的挂牌企业; (2) 剔除ST和*ST 类上市公司; （3）剔除样本期内财务数据不完整的企业; （4）剔除样本期间内无融资行为 
的挂牌企业。本文最终选取了 705家符合条件的样本企业作为研究对象, 财务数据及相关指标 均来源于Choice金融数据库。

\subsection{2 数据处理}

运用DEA模型的前提条件是决策单元的各项投入产出指标均为正数, 而在企业融资效率 的实际测算中, 净利润和净资产收益率等相关指标不可避免的出现负数。因此, 需要提前对 样本数据做无量纲化处理。本文采用功能系数法将原始数据全部正向标准化, 在不改变数据 间逻辑关系的基础上将其归集在0-1的区间内。数据处理公式如下:

$$
X_{i j}^{*}=0.1+0.9 \times \frac{X_{i j}-m_{j}}{M_{j}-m_{j}}
$$

其中: $X_{i j}$ 为变量原始值, $M_{j}=\max \left(X_{i j}\right), m_{j}=\min \left(X_{i j}\right), X_{i j}^{*}=[0,1]$ 。

\section{4. 实证分析}

\section{1 描述性统计}

在测度样本企业融资效率之前，本文先对各项样本指标进行描述性统计分析，从均值、 中值和方差等不同角度去刻画指标的基本统计特征。观察表2的描述性统计结果，可以发现新 三板市场中的科技型中小企业具有以下特点。第一，企业的资产规模不断扩张，且样本企业 个体之间在资产规模方面差异显著。第二，行业的资产负债率下降明显，后期整体维持在 $33 \%$ 左右较低的水平。这是由于企业在新三板市场融资后，股权资金比例的提高有效降低了企业 的资产负债率。第三，企业对于资产的管理效率不高，主要体现在行业的净利润均指在2016 年出现明显下滑且逐年下降, 净资产收益率更是在样本期内持续降低, 反映融资后的科技型 企业其盈利能力并没有获得提升。第四, 行业的自主创新研发能力不足, 主要表现在样本企 业的无形资产增加额在观察期内的中值长期为负。

表2 样本指标的描述性统计分析

\begin{tabular}{|c|c|c|c|c|c|c|c|c|}
\hline 年度 & 统计量 & $\begin{array}{c}\text { 资产总额 } \\
\text { (万元) }\end{array}$ & $\begin{array}{c}\text { 资产负债率 } \\
(\%)\end{array}$ & $\begin{array}{c}\text { 营业总成本 } \\
\text { (万元) }\end{array}$ & $\begin{array}{l}\text { 净利润 } \\
\text { (万元） }\end{array}$ & $\begin{array}{c}\text { 净资产收益 } \\
\text { 率 }(\%) \text {. }\end{array}$ & $\begin{array}{c}\text { 营业总收入 } \\
\text { (万元） }\end{array}$ & \begin{tabular}{|l} 
无形资产增 \\
加额 (万元)
\end{tabular} \\
\hline \multirow{5}{*}{2014} & 均值 & 12362.80 & 41.11 & 11000.38 & 864.04 & 16.84 & 11697.48 & 59.56 \\
\hline & 中值 & 6336.14 & 39.65 & 4585.91 & 363.38 & 13.56 & 5009.63 & -0.93 \\
\hline & 标准差 & 20967.09 & 21.31 & 52805.81 & 1750.90 & 29.87 & 54152.26 & 436.27 \\
\hline & 极小值 & 285.06 & 1.42 & 186.26 & -3904.09 & -271.96 & 24.65 & -2269.15 \\
\hline & 极大值 & 290859.79 & 99.97 & 1325583.11 & 24086.69 & 313.42 & 1357826.16 & 4691.19 \\
\hline \multirow{5}{*}{2015} & 均值 & 17150.77 & 32.53 & 13092.57 & 1171.17 & 15.39 & 14033.44 & 122.40 \\
\hline & 中值 & 9280.28 & 30.74 & 5792.87 & 627.00 & 14.04 & 6379.85 & -1.63 \\
\hline & 标准差 & 29556.51 & 18.76 & 62085.51 & 2503.72 & 20.82 & 63461.43 & 614.74 \\
\hline & 极小值 & 577.62 & 1.07 & 310.82 & -7319.18 & -83.20 & 87.33 & -1498.86 \\
\hline & 极大值 & 347926.33 & 98.12 & 1554705.67 & 33279.16 & 129.28 & 1587087.99 & 7538.45 \\
\hline \multirow{5}{*}{2016} & 均值 & 21077.93 & 32.59 & 15536.18 & 1172.11 & 8.60 & 16447.06 & 161.11 \\
\hline & 中值 & 11114.41 & 30.06 & 7090.91 & 594.83 & 9.70 & 7874.55 & -1.01 \\
\hline & 标准差 & 37961.53 & 18.12 & 66636.20 & 3306.14 & 19.31 & 68101.67 & 674.91 \\
\hline & 极小值 & 319.85 & 0.61 & 372.80 & \begin{tabular}{|l|}
-10449.39 \\
\end{tabular} & -90.74 & 24.68 & -2096.92 \\
\hline & 极大值 & 427124.61 & 97.83 & 1640137.49 & 50384.90 & 74.60 & 1671030.19 & 7087.26 \\
\hline \multirow{5}{*}{2017} & 均值 & 23353.24 & 33.10 & 17228.90 & 1039.16 & 5.27 & 17947.07 & 97.54 \\
\hline & 中值 & 12801.56 & 30.69 & 7919.90 & 537.77 & 7.61 & 8166.99 & -4.70 \\
\hline & 标准差 & 41310.02 & 18.38 & 63607.51 & 4619.65 & 21.87 & 65093.74 & 770.06 \\
\hline & 极小值 & 447.35 & 0.39 & 265.95 & -16344.17 & -113.20 & 19.62 & -6754.19 \\
\hline & 极大值 & 494418.38 & 97.64 & 1496553.85 & 89048.66 & 99.88 & 1523460.80 & 8973.71 \\
\hline 2018 & 均值 & 25157.26 & 33.45 & 19176.86 & 826.14 & 2.07 & 19679.69 & 55.72 \\
\hline
\end{tabular}




\begin{tabular}{|c|c|c|c|c|c|c|c|c|}
\hline \multirow{7}{*}{} & 中值 & 13229.90 & 32.27 & 8538.59 & 341.68 & 5.09 & 8653.89 & -7.81 \\
\cline { 2 - 8 } & 标准差 & 45674.65 & 18.69 & 71010.08 & 5495.54 & 22.09 & 73134.24 & 599.73 \\
\cline { 2 - 8 } & 极小值 & 251.35 & 0.14 & 277.64 & -28702.93 & -85.52 & 96.60 & -5008.44 \\
\cline { 2 - 9 } & 极大值 & 564475.14 & 98.37 & 1627521.12 & 78474.75 & 103.03 & 1656699.26 & 6636.31 \\
\hline
\end{tabular}

\subsection{DEA-BCC 模型下融资效率静态分析}

本文基于DEA-BCC模型，运用DEAP2.1软件对705家科技型中小企业在2014-2018年间的 融资效率进行测算, 分别得到不同年份的综合效率值、纯技术效率值和规模效率值, 数据整 理后的结果见表3。

表3 2014-2018年样本企业融资效率统计结果

\begin{tabular}{|c|c|c|c|c|c|c|c|c|}
\hline \multirow{2}{*}{ 年份 } & \multirow{2}{*}{ 效率类型 } & \multirow{2}{*}{ 均值 } & \multicolumn{2}{|c|}{ 有效 } & \multicolumn{2}{|c|}{ 相对有效 } & \multicolumn{2}{|c|}{ 相对无效 } \\
\hline & & & 家数 & 占比 & 家数 & 占比 & 家数 & 占比 \\
\hline \multirow{3}{*}{2014} & 综合技术效率 & 0.984 & 25 & $3.55 \%$ & 680 & $96.45 \%$ & 0 & $0.00 \%$ \\
\hline & 纯技术效率 & 0.988 & 46 & $6.52 \%$ & 659 & $93.48 \%$ & 0 & $0.00 \%$ \\
\hline & 规模效率 & 0.996 & 51 & $7.23 \%$ & 654 & $92.77 \%$ & 0 & $0.00 \%$ \\
\hline \multirow{3}{*}{2015} & 综合技术效率 & 0.977 & 34 & $4.82 \%$ & 671 & $95.18 \%$ & 0 & $0.00 \%$ \\
\hline & 纯技术效率 & 0.984 & 41 & $5.82 \%$ & 664 & $94.18 \%$ & 0 & $0.00 \%$ \\
\hline & 规模效率 & 0.993 & 81 & $11.49 \%$ & 624 & $88.51 \%$ & 0 & $0.00 \%$ \\
\hline \multirow{3}{*}{2016} & 综合技术效率 & 0.976 & 31 & $4.40 \%$ & 674 & $95.60 \%$ & 0 & $0.00 \%$ \\
\hline & 纯技术效率 & 0.982 & 43 & $6.10 \%$ & 662 & $93.90 \%$ & 0 & $0.00 \%$ \\
\hline & 规模效率 & 0.994 & 119 & $16.88 \%$ & 586 & $83.12 \%$ & 0 & $0.00 \%$ \\
\hline \multirow{3}{*}{2017} & 综合技术效率 & 0.972 & 25 & $3.55 \%$ & 680 & $96.45 \%$ & 0 & $0.00 \%$ \\
\hline & 纯技术效率 & 0.977 & 33 & $4.68 \%$ & 672 & $95.32 \%$ & 0 & $0.00 \%$ \\
\hline & 规模效率 & 0.995 & 167 & $23.69 \%$ & 538 & $76.31 \%$ & 0 & $0.00 \%$ \\
\hline \multirow{3}{*}{2018} & 综合技术效率 & 0.971 & 24 & $3.40 \%$ & 681 & $96.60 \%$ & 0 & $0.00 \%$ \\
\hline & 纯技术效率 & 0.976 & 34 & $4.82 \%$ & 671 & $95.18 \%$ & 0 & $0.00 \%$ \\
\hline & 规模效率 & 0.995 & 157 & $22.27 \%$ & 548 & $77.73 \%$ & 0 & $0.00 \%$ \\
\hline
\end{tabular}

观察表中数据可以发现，2014-2018年间我国科技型中小企业融资综合技术效率值为 1 的企业每年占比不足 $5 \%$, 且在样本期内融资有效的企业比例呈现先增长后下降的趋势, 表明 我国新三板市场中科技型中小企业融资效率整体水平不高, 仅有很少一部分企业融资能够同 时达到技术有效和规模有效的状态, 大部分科技型企业融资效率未达到DEA有效且未出现效 率改善的趋势。

$\mathrm{BBC}$ 模型下，企业融资的综合效率受到纯技术效率和规模效率的共同影响。观察效率指 标的均值可以发现，2014-2018年间综合技术效率均值均小于纯技术效率和规模效率的均值， 规模效率均值在样本期间内波动很小, 基本稳定在 0.995 上下, 达到规模有效的企业占比逐年 提高, 这表明规模效率并不是影响科技型企业融资综合效率较低的主要原因。企业融资的纯 技术效率均值一直低于规模效率的均值, 且达到纯技术效率有效的企业家数逐年降低, 说明 代表公司内部管理水平的纯技术效率低下是导致科技型企业融资效率整体水平不高的主要原 因。

\subsection{DEA-Malmquist模型下融资效率动态分析}

为了更准确地反应新三板市场中科技型中小企业融资效率的动态变化, 本文在对融资效 率静态分析的基础上, 引入Malmquist指数进一步测算该行业融资效率的动态变化情况。由于 DEA-Malmquist指数将全要素生产率变动分解为技术效率变动和技术进步, 技术效率变动可 进一步分解为纯技术效率和规模效率, 从而更为深入系统地分析影响企业融资效率变动的内 
在机理。通过对705家样本企业在2014-2018年间的融资效率进行动态分析, 得出如下表4.3所 示的统计结果。

表4 2014-2018年Malmquist指数统计结果

\begin{tabular}{|c|c|c|c|c|c|}
\hline \multirow{2}{*}{ 年份 } & 技术效率变动 & 技术进步 & 纯技术效率变动 & 规模效率变动 & 全要素生产率变动 \\
\cline { 2 - 6 } & (effch) & (techch) & (pech) & (sech) & (tfpch) \\
\hline $2014-2015$ & 0.993 & 1.005 & 0.996 & 0.997 & 0.998 \\
\hline $2015-2016$ & 0.999 & 0.997 & 0.997 & 1.002 & 0.996 \\
\hline $2016-2017$ & 0.996 & 1.001 & 0.996 & 1.001 & 0.997 \\
\hline $2017-2018$ & 0.998 & 0.998 & 0.999 & 1 & 0.996 \\
\hline 几何均值 & 0.997 & 1 & 0.997 & 1 & 0.997 \\
\hline
\end{tabular}

从整体上看, 科技型中小企业在2014-2018年的全要素生产率变动指数均值为 0.997, 且每 年的变动指数均小于 1 , 表明行业整体融资效率在观察期内呈小幅下降趋势。观察发现, 样本 期内技术进步指数均值为 1 , 高于技术效率变动均值, 说明技术效率退步是导致融资效率下降 的主要原因。对于技术效率变动进一步分析发现, 纯技术效率变动和规模效率变动共同影响 着其变化。规模效率变动指数在 2015-2018年分别为 $1.002 、 1.001$ 和 1 , 说明企业的投入产出 规模已达到最佳生产效益的合理范围，造成技术效率出现退步的原因是纯技术效率的下降， 公司内部管理体制存在不足对融资效率产生负面影响, 这与静态分析的结论基本一致。通过 观察2014-2018年效率指标的变化趋势可以发现, 全要素生产率与技术进步指标变动趋势保 持同步, 恰好说明科技型企业融资效率对于技术进步的敏感度较高, 技术进步是切实提高融 资效率的根本动力。

\section{4 融资效率影响因素分析}

为了进一步探究科技型中小企业融资效率的影响因素，本文选取Tobit计量经济模型，从 企业的融资结构、盈利能力、营运能力和发展能力四个方面出发探究对融资效率的影响。模 型最终选择以DEA测算的融资综合技术效率值（TE）作为因变量，以资产负债率（DEBT）、 净资产收益率 (ROE) 、总资产周转率 (ATT) 和营业收入增长率 (POP) 为自变量, 以企 业规模 (SIZE) 和年份 (YEAR) 为控制变量进行多元回归分析, 具体模型构建如下: $T E_{i, t}=\alpha+\beta_{1} \times D E B T+\beta_{2} \times R O E+\beta_{3} \times A T T+\beta_{4} \times P O P+\beta_{5} \times S I Z E+\beta_{6} \times Y E A R_{1}+\beta_{7} \times Y E A R_{2}+\beta_{8} \times Y E A R_{3}+\varepsilon$

其中, $\alpha$ 表示截距项， $\beta$ 表示该自变量与因变量之间的相关系数， $\varepsilon$ 代表随机扰动项。

运用Eviews9.0对2014-2018我国新三板市场中科技型中小企业融资效率影响因素进行 Tobit回归, 模型处理结果如表5所示。

表5 Tobit模型回归结果

\begin{tabular}{|c|c|c|}
\hline 变量 & 系数 & P值 \\
\hline DEBT & $-0.0005 * * *$ & 0.0000 \\
\hline ROE & $0.0002 * * *$ & 0.0000 \\
\hline ATT & $0.0144 * * *$ & 0.0000 \\
\hline POP & 0.0000 & 0.5037 \\
\hline SIZE & $0.0522 * * *$ & 0.0000 \\
\hline YEAR1 & $0.0347 * * *$ & 0.0000 \\
\hline YEAR2 & $0.0213 * * *$ & 0.0000 \\
\hline YEAR3 & $0.0153 * * *$ & 0.0000 \\
\hline
\end{tabular}

注: *表示 $10 \%$ 的水平下显著, $* *$ 表示 $5 \%$ 的水平下显著, $* * *$ 表示 $1 \%$ 的水平下显著。

实证结果表明: 
（1）在0.01的显著水平上，企业的资产负债率与融资效率呈负相关，即企业的债务水平 越高, 融资效率越低。这是由于债务融资比例的提高会增加中小企业的偿债风险, 使得企业 迫于还款压力和资金的不确定性降低对融入资金的使用效率。因此，企业应合理控制公司的 融资结构, 适度降低企业的资产负债率和财务杜杆水平对企业融资效率的提升具有促进作用。

（2）企业的净资产收益率与融资效率呈正相关关系，即企业的盈利能力对融资效率具有 正向作用。因此，企业应加大自主创新力度，努力研发高新技术培养企业的核心竞争力，通 过提升公司的盈利能力促进融资效率的提高。

(3) 企业的总资产周转率与融资效率呈正相关关系, 且影响显著, 即企业的营运能力对 融资效率的提高具有促进作用。企业通过提升公司的经营管理水平，加快资金的周转速度， 实现对于资源的充分有效利用，从而切实提升公司的融资效率。

（4）企业的营业收入增长率与融资效率之间并不存在显著的相关关系，即企业的发展能 力并未对融资效率产生明显影响。这可能是由于新三板中小企业的融资大多具有盲目性, 较 多企业的融资行为仅是为了提高资金的流动性，与公司战略制定或项目融资关系较小。

\section{5. 研究结论及建议}

本文通过将DEA模型与Malmquist指数和Tobit计量分析模型有机结合, 构建了符合行业特 征的评价指标体系，对2014-2018年我国新三板市场中科技型中小企业融资效率进行静态和 动态分析, 并对融资效率的影响因素展开研究。实证研究结论如下：（1）我国科技型中小企 业融资效率整体水平不高, 规模效率表现较好, 纯技术效率较低是制约融资效率不高的主要 原因。（2）通过对融资效率的动态分析发现，科技型中小企业全要素生产率在样本期内呈小 幅下降趋势，技术效率退步是导致融资效率下降的主要原因，而纯技术效率下降是技术效率 退步的主要因素，技术进步是切实提高融资效率的原动力。（3）回归分析发现，企业的资产 负债率会抑制融资效率的提高, 企业的盈利能力和营运能力对融资效率的提升具有正向促进 作用，企业的发展能力并不能对融资效率产生明显影响。

基于上述研究结果，本文针对科技型中小企业融资效率的提高提出以下建议：一是科技 型企业应努力提高自主创新能力, 加大研发投入力度, 培养、引进高新技术人才, 学习吸收 先进生产技术，培养企业的核心竞争力，通过技术进步有效提升企业的融资效率。二是优化 企业的融资结构, 适当降低企业的负债水平，选择优先股或可转债等多种方式实现多元化融 资, 从而降低企业的债务负担和融资风险, 相应使得企业融资效率获得提高。三是注重提升 企业的内部经营管理水平，完善内部控制制度和公司治理结构，通过科学的管理体制实现对 资源的高效利用，从而提升企业的纯技术效率水平，实现企业融资效率的提高。

\section{References}

[1] Zeng Kanglin. How to Treat Direct and Indirect Financing[J]. Financial Research, 1993(10): 7-11.

[2] Song Wenbing. Several Issues Need to Be Clarified on Financing Methods [J]. Financial Research, 1998(1): 35-42.

[3] Cheng Shouhong. How to Evaluate the Financing Efficiency of Small and Medium Enterprises[J].Financial and Accounting Monthly, 2003(14):21.

[4] Gao Xuezhe. Corporate Finance Efficiency: Connotation and Extension[J]. Productivity Research, 2005(6).

[5] Zhu Bingxin.Fuzzy Comprehensive Evaluation and Empirical Analysis of Zhejiang SMEs' Financing Efficiency[J].Zhejiang Statistics,2005(10):14-16. 
[6] Wang Tao. An Empirical Study on the Financing Efficiency of the New Third Board Concept Stocks [D]. Anhui University, 2014.

[7] Li Xin. Dynamic Research on Financing Efficiency of SMEs_-Based on DEA-Malmquist Index Method[J].Accounting Newsletter,2015(26):34-36.

[8] Xiu Guoyi, Li Daizhe. Research on the Measurement Efficiency of New Third Board of Science and Technology SMEs[J].Science \& Technology Progress and Policy,2016,33(14): 124-128.

[9] Shen Chen, A Research on Financing Efficiency of SMEs in NEEQ Market:Private Placement Based on Three Stage DEA Model[J]. Audit and Economic Research, 2017, 32(03): 78-86.

[10] Feng Taozhu, Li Jingxuan. Research on Financing Efficiency of Military Listed Companies under the Perspective of Military-Military Integration_—Based on Two-stage Chained DEA Model[J].Friends of Accounting, 2019(06):77-82.

[11] Wang Xuemei,Jia Qiqi.Study on the Financing Efficiency of China's Manufacturing SMEs Based on DEA-Malmquist Index[J].Wuhan Finance,2018(08):56-61.

[12] Sun Yanfang,Cao Yongpeng.Analysis of Financing Efficiency of Public Utilities Listed Companies[J].Shandong Social Sciences,2018(09):168- 173.

[13] Song Guanghui,Li Hongfa,Xu Lin.Study on Financing Efficiency of SMEs Based on Two-stage DEA[J].Science and Technology Management Research, 2017,37(02):191-195.

[14] Xu Kai. Analysis on the Financing Efficiency of Listed Enterprises in China's New Third Board Market[J].New Finance,2018(04):50-56.

[15] Ding Hua, Gao Dan. Financing Efficiency of New Third Board Listing Enterprises_—Based on DEA-Malmquist Method[J].Friends of Accounting, 2019(02): 21-25.

[16] Xiao Ya,Guo Xiaoshun.Evaluation of Equity Financing Efficiency of New Third Board High-tech Enterprises[J].Accounting Monthly,2018(11):57-61.

[17] Wang Jianjun, Qiu Jie, Yu Qitong.Measurement of Financing Efficiency of China's Manufacturing Listed Companies in Small and Medium-sized Board Market_-Based on Three-stage DEA-Malmquist Model[J].Contemporary Finance Research,2018(05):68-80. 